\title{
On the Evaluation of Some Integrals Occurring in Scattering Problems
}

\author{
By J. Kadlec
}

\begin{abstract}
Some definite integrals which occur in transport problems through a scattering medium are studied. They are expressed in terms of such functions as the exponential integral of the first and second order, the dilogarithm, and a newly introduced and tabulated function.
\end{abstract}

1. Introduction. Definite integrals of the type

$$
\int_{0}^{1} d x Q(x) \prod_{i=1}^{q}\left(a_{i} x+b_{i}\right)^{-1} f(x), \quad a_{i} \neq 0,
$$

where $Q(x)$ is a real polynomial in $x$ of order $p(p \leqslant q)$, and $f(x)$ is one of the functions $e^{-\gamma / x}, \ln |m x+n|, \ln |m x+n| \cdot e^{-\gamma / x}$ or the exponential integral $E_{1}(m / x+n)$, occur in various transport problems involving scattering (e.g. penetration of particles through a solid or scattering of the light in a planetary atmosphere). The author has met them while calculating the scattering of excited electrons in thin metal films [4].

The straightforward numerical integration in (1) can be rather difficult because of the possible logarithmic singularity and/or possible poles at $x_{i}=-b_{i} / a_{i}$ in the integrand. Moreover, it is sometimes desirable to possess a closed solution of the above integral. The purpose of this paper is to present such formulae which can be used as an expedient for calculations in scattering problems. To obtain clearly tractable results, we perform the whole calculation in terms of principal parts of the individual functions. It means that the expressions of the type $\ln |u| \cdot \ln |v|$ and $\ln |u| \cdot E_{1}(v)$ in the resulting formulae are determined up to the additive constant $\pi^{2}$ which must be considered in particular cases.

The integrand of the integral (1) can be written in the form $\left(A_{0}, A_{i}\right.$ are constants):

$$
Q(x) \prod_{i=1}^{q}\left(a_{i} x+b_{i}\right)^{-1} f(x)=A_{0} f(x)+\sum_{i=1}^{q} A_{i}\left(a_{i} x+b_{i}\right)^{-1} f(x) .
$$

Consequently, the evaluation of (1) reduces to the calculation of integrals of the type $\int_{0}^{1} d x f(x)$, and $\int_{0}^{1} d x(a x+b)^{-1} f(x)$. These integrals lead to formulae which contain besides the familiar functions also less-well-known higher functions such as exponential integral of the first order $E_{1}(x)$, exponential integral of the second order $E_{1}^{(2)}(x)$, and

Received April 15, 1975; revised August 25, 1975.

AMS (MOS) subject classifications (1970). Primary 26A63; Secondary 30A86, 33A70.

Key words and phrases. Mathematics of scattering problems, exponential integrals, dilogarithm, definite integrals. 
dilogarithm $L_{2}(x)$. All these functions are generally defined for a complex variable, but we will use them only for real values of $x$.

The exponential integral of the first order $E_{1}(x)$ is defined on the real axis by [1], $[5],[7]:$

$$
E_{1}(-x)=-E i(x), \quad E i(x)=\int_{-\infty}^{x} d t t^{-1} e^{t}, \quad-\infty<x<\infty,
$$

and the integral is interpreted in the sense of the Cauchy principal value if $x>0$. For practical purposes we note:

$$
E_{1}(x)=\int_{x}^{\infty} d t t^{-1} e^{-t}=\int_{1}^{\infty} d t t^{-1} e^{-x t} .
$$

The values of $E_{1}(x)$ and/or $E i(x)$ are tabulated or they can be calculated up to the desired accuracy from various approximations [1], [7] .

The exponential integral of the second order $E_{1}^{(2)}(x)$ has been introduced and tabulated by van de Hulst [3] (see also [5], [7]):

$$
E_{1}^{(2)}(x)=\int_{x}^{\infty} d t t^{-1} E_{1}(t)=\int_{1}^{\infty} d t t^{-1} \ln t e^{-x t}, \quad x>0 .
$$

For the dilogarithm $L_{2}(x)$ we take the standard form [6], [8] :

$$
L_{2}(x)=-\int_{0}^{x} d t t^{-1} \ln |1-t|, \quad-\infty<x<\infty .
$$

An extensive table $(\Delta x=0.001)$ and some properties of $L_{2}(x)$ can be found in Mitchell's paper [8].

For the sake of clarity the functions $E_{1}(x), E_{1}^{(2)}(x)$, and $L_{2}(x)$ are shown in Fig. 1.

The following identity will often be used.

$$
[x(a x+b)]^{-1}=(b x)^{-1}-a[b(a x+b)]^{-1} .
$$

The calculation of (1) involves no unusual steps. Substitution and integration by parts are frequently applied. The integration can, in general, be performed in a closed form only for the case when the function $f(x)$ is $e^{-\gamma / x}$ or $\ln |m x+n|$. If $f(x)$ is $\ln |m x+n| \cdot e^{-\gamma / x}$ or $E_{1}(m / x+n)$, it is necessary to introduce a new function of three variables $\operatorname{Le}(A, B, \gamma)$ (see (16)), the evaluation of which is described in Section 6.

2. Integrals $\int_{0}^{1} d x e^{-\gamma / x}$ and $\int_{0}^{1} d x(a x+b)^{-1} e^{-\gamma / x}$. The first integral can be solved by the substitution $x=t^{-1}$ and subsequent integration by parts; using (4), we obtain

$$
\int_{0}^{1} d x e^{-\gamma / x}=e^{-\gamma}-\gamma E_{1}(\gamma), \quad \gamma>0 .
$$

In the second integral of this group we substitute $x=u^{-1}$, use relation (7), substitute $t=\gamma(u+a / b)$ in the second term, and then apply (4). Thus,

$$
\int_{0}^{1} d x(a x+b)^{-1} e^{-\gamma / x}=a^{-1}\left\{E_{1}(\gamma)-e^{\gamma a / b} E_{1}(\gamma+\gamma a / b)\right\}
$$

$$
\gamma>0, a \neq 0 \text {. }
$$


(In the case $b=0$, the right-hand side of (9) reduces to the first term because $\lim _{b \rightarrow 0} e^{\gamma a / b} E_{1}(\gamma+\gamma a / b)=0$; see [5].)

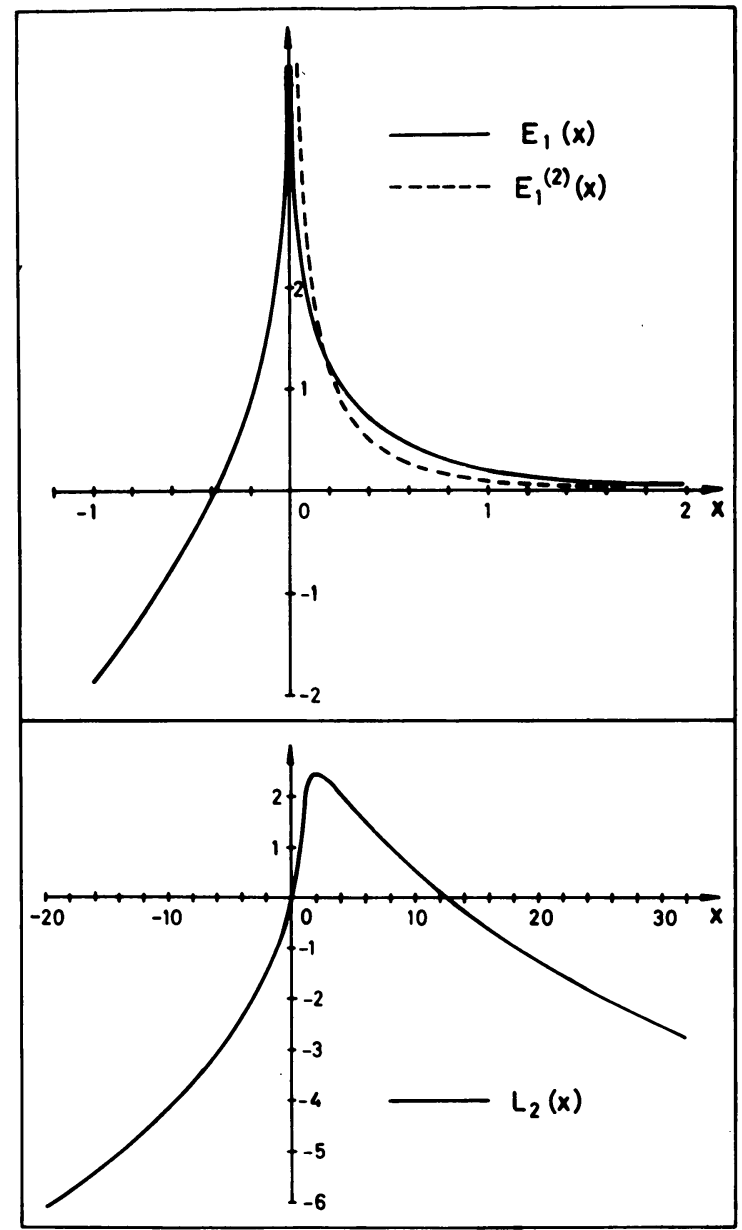

FIGURE 1. Exponential integral of the first order $E_{1}(x)$ and of the second $\operatorname{order} E_{1}^{(2)}(x)$ (above) and the dilogarithm $L_{2}(x)$ (below).

3. Integrals $\int_{0}^{1} d x \ln |m x+n|$ and $\int_{0}^{1} d x(a x+b)^{-1} \ln |m x+n|$. For the evaluation of the first integral, we have

$$
\int_{0}^{1} d x \ln |m x+n|=m^{-1}\{(m+n)(\ln |m+n|-1)-n(\ln |n|-1)\}
$$

$$
m \neq 0 \text {. }
$$

Using the indefinite integral given in [8], we obtain for the second integral,

$$
\begin{aligned}
& \int_{0}^{1} d x \frac{\ln |m x+n|}{a x+b} \\
& =a^{-1}\left\{\ln \left|\frac{m b-n a}{a}\right| \ln \left|\frac{a+b}{b}\right|-L_{2}\left(\frac{m(a+b)}{m b-n a}\right)+L_{2}\left(\frac{m b}{m b-n a}\right)\right\}, \\
& \quad a \neq 0, \quad b \neq 0, \quad m \neq 0,
\end{aligned}
$$

where $L_{2}(y)$ is the dilogarithm defined by (6). 
4. Integrals $\int_{0}^{1} d x \ln |m x+n| e^{-\gamma / x}$ and $\int_{0}^{1} d x(a x+b)^{-1} \ln |m x+n| e^{-\gamma / x}$. In view of the result in [8], we integrate by parts in the indefinite integral

$$
\begin{aligned}
\int d x \frac{\ln |m x+n| e^{-\gamma x}}{a x+b} & =a^{-1}\left\{\ln \left|\frac{m b-n a}{a}\right| \ln \left|\frac{m(a x+b)}{m b-n a}\right|-L_{2}\left(\frac{m(a x+b)}{m b-n a}\right)\right\} e^{-\gamma x} \\
& +\gamma a^{-1} \int d x\left\{\ln \left|\frac{m b-n a}{a}\right| \ln \left|\frac{m(a x+b)}{m b-n a}\right|\right. \\
& \left.-L_{2}\left(\frac{m(a x+b)}{m b-n a}\right)\right\} e^{-\gamma x}+\text { const. }
\end{aligned}
$$

It can be seen that the solution of the integral on the right in (12) reduces to the evaluation of the integrals $\int d x e^{-\gamma x}, \int d x \ln |a x+b| e^{-\gamma x}$, and

$$
\int d x L_{2}(m(a x+b) /(m b-n a)) e^{-\gamma x} .
$$

The first one is trivial. The second integral on substituting $a x+b=y$ takes the form $\int d y \ln |y| e^{\lambda y}$ which equals $\lambda^{-1}\left\{\ln |y| e^{\lambda y}+E_{1}(-\lambda y)\right\}$ as given in [2]. Substituting back for $y$, we obtain

$$
\begin{aligned}
\int d x \ln \mid a x & +b \mid e^{-\gamma x} \\
= & -\gamma^{-1}\left\{e^{-\gamma x} \ln |a x+b|+e^{\gamma b / a} E_{1}(\gamma x+\gamma b / a)\right\}+\text { const. }
\end{aligned}
$$

Then the integral (12) reads

$$
\begin{aligned}
& \int d x \frac{\ln |m x+n| e^{-\gamma x}}{a x+b} \\
& \text { (14) }=-a^{-1}\left\{L_{2}\left(\frac{m(a x+b)}{m b-n a}\right) e^{-\gamma x}+e^{\gamma b / a} \ln \left|\frac{m b-n a}{a}\right| E_{1}(\gamma x+\gamma b / a)\right. \\
&\left.+\gamma \int d x L_{2}\left(\frac{m(a x+b)}{m b-n a}\right) e^{-\gamma x}\right\}+ \text { const. }
\end{aligned}
$$

In particular,

$$
\begin{aligned}
& \int_{1}^{\infty} d x \frac{\ln |m x+n| e^{-\gamma x}}{a x+b} \\
&=a^{-1}\left\{L_{2}\left(\frac{m(a+b)}{m b-n a}\right) e^{-\gamma}+e^{\gamma b / a} \ln \left|\frac{m b-n a}{a}\right| E_{1}(\gamma+\gamma b / a)\right. \\
&\left.-\gamma \operatorname{Le}\left(\frac{m a}{m b-n a}, \frac{m b}{m b-n a}, \gamma\right)\right\}
\end{aligned}
$$

where we have introduced a new function $\operatorname{Le}(A, B, \gamma), \gamma>0$, defined by

$$
\operatorname{Le}(A, B, \gamma)=\int_{1}^{\infty} d x L_{2}(A x+B) e^{-\gamma x}
$$

The function $\operatorname{Le}(A, B, \gamma)$ is evaluated in Section 6 .

We now consider the calculation of $\int_{0}^{1} d x \ln |m x+n| e^{-\gamma / x}$. Substituting $x=$ $t^{-1}$, we have 
(17) $\int_{0}^{1} d x \ln |m x+n| e^{-\gamma / x}=\int_{1}^{\infty} d t t^{-2} \ln |n t+m| e^{-\gamma t}-\int_{1}^{\infty} d t t^{-2} \ln t e^{-\gamma t}$.

The first integral on the right can be integrated by parts, giving the integral solved in (9) together with an integral of the type (15). The second integral splits similarly into the type (8) and the exponential integral of the second order $E_{1}^{(2)}(\gamma)$ defined by (5). Consequently, we write the integral (17) in the form

$\int_{0}^{1} d x \ln |m x+n| e^{-\gamma / x}=\ln |m+n| e^{-\gamma}+[n / m-\gamma \ln |m|+\gamma] E_{1}(\gamma)-e^{-\gamma}$

$$
\begin{aligned}
& -n / m e^{\gamma m / n} E_{1}(\gamma+\gamma m / n)-\gamma L_{2}(-n / m) e^{-\gamma} \\
& +\gamma E_{1}^{(2)}(\gamma)+\gamma^{2} \operatorname{Le}(-n / m, 0, \gamma), \quad \gamma>0, m \neq 0 .
\end{aligned}
$$

In particular, if $n=0$,

$$
\begin{array}{r}
\int_{0}^{1} d x \ln |m x| e^{-\gamma / x}=[\ln |m|-1]\left[e^{-\gamma}-\gamma E_{1}(\gamma)\right]+\gamma E_{1}^{(2)}(\gamma) \\
\gamma>0, \quad m \neq 0 .
\end{array}
$$

The integral $\int_{0}^{1} d x(a x+b)^{-1} \ln |m x+n| e^{-\gamma / x}$ converts by substituting $x=t^{-1}$ and applying (7) into three integrals of the type (15) and the function (5). After some calculation we obtain

$$
\begin{aligned}
& \int_{0}^{1} d x \frac{\ln |m x+n| e^{-\gamma / x}}{a x+b} \\
& =a^{-1}\left\{\ln |m| E_{1}(\gamma)-e^{\gamma a / b} \ln \left|\frac{n a-m b}{a}\right| E_{1}(\gamma+\gamma a / b)\right. \\
& +\left[L_{2}\left(-\frac{n}{m}\right)-L_{2}\left(\frac{n(a+b)}{n a-m b}\right)+L_{2}\left(\frac{a+b}{a}\right)\right] e^{-\gamma}-E_{1}^{(2)}(\gamma) \\
& \left.-\left[\operatorname{Le}\left(-\frac{n}{m}, 0, \gamma\right)-\operatorname{Le}\left(\frac{n b}{n a-m b}, \frac{n a}{n a-m b}, \gamma\right)+\operatorname{Le}\left(\frac{b}{a}, 1, \gamma\right)\right]\right\} \text {, } \\
& \gamma>0, a \neq 0, m \neq 0 \text {. }
\end{aligned}
$$

(For $b=0$, see the note at (9); if $m=0$, (20) becomes (9); if $a=0,(20)$ becomes (18).)

5. Integrals $\int_{0}^{1} d x E_{1}(m / x+n)$ and $\int_{0}^{1} d x(a x+b)^{-1} E_{1}(m / x+n)$. To evaluate $\int_{0}^{1} d x E_{1}(m / x+n)$, we proceed as follows. The substitution $x=t^{-1}$ converts this integral into $\int_{1}^{\infty} d t t^{-2} E_{1}(m t+n)$. Integrating by parts and then using the identity (7) and relation (4), we have

$$
\begin{aligned}
\int_{0}^{1} d x E_{1}(m / x+n)=n^{-1}\left\{[m+n] E_{1}(m+n)-m e^{-n} E_{1}(m)\right\}, & \\
& m \neq 0, \quad n \neq 0 .
\end{aligned}
$$

(For $n=0,(21)$ becomes (8) after one integration by parts.)

In the second integral of this group we substitute $x=t^{-1}$ again and apply relation

(7). In this way the problem reduces to the evaluation of $\int_{1}^{\infty} d t t^{-1} E_{1}(m t+n)$ and 
$\int_{1}^{\infty} d t(b t+a)^{-1} E_{1}(m t+n)$. Integrating now by parts and using (15), we obtain

$$
\int_{0}^{1} d x \frac{E_{1}(m / x+n)}{a x+b}
$$

$$
\begin{array}{r}
=a^{-1} e^{-n}\left\{e^{-m}\left[L_{2}\left(\frac{m+n}{n}\right)-L_{2}\left(\frac{b(m+n)}{n b-m a}\right)\right]-e^{n} \ln \left|\frac{n b-m a}{n(a+b)}\right| E_{1}(m+n)\right. \\
\left.-m\left[\operatorname{Le}\left(\frac{m}{n}, 1, m\right)-\operatorname{Le}\left(\frac{m b}{n b-m a}, \frac{n b}{n b-m a}, m\right)\right]\right\} \\
m>0, n \neq 0, a \neq 0 .
\end{array}
$$

(For $n=0,(22)$ can be evaluated using (5) and (15).)

6. Evaluation of $\operatorname{Le}(A, B, \gamma)=\int_{1}^{\infty} d x L_{2}(A x+B) e^{-\gamma x}$. In (16) of Section 4 we have introduced the function $\operatorname{Le}(A, B, \gamma)$ of three variables $A, B, \gamma$. This function converges always for $\gamma>0$ and real $A, B$. In particular, if $A=0$,

$$
\operatorname{Le}(0, B, \gamma)=L_{2}(B) \gamma^{-1} e^{-\gamma}
$$

Let $A \neq 0, \gamma>0$. The substitution $A x+B=y$ in (16) leads to the function $\operatorname{Le}^{*}(\alpha, \kappa)$ defined by

$$
\operatorname{Le}^{*}(\alpha, \kappa)= \begin{cases}\int_{\alpha}^{\infty} d y L_{2}(y) e^{\kappa(\alpha-y)}, & \kappa>0, \\ \int_{\alpha}^{-\infty} d y L_{2}(y) e^{\kappa(\alpha-y)}, & \kappa<0 .\end{cases}
$$

Then

$$
\operatorname{Le}(A, B, \gamma)=A^{-1} e^{-\gamma} \operatorname{Le}^{*}(A+B, \gamma / A)
$$

The function $\mathrm{Le}^{*}(\alpha, \kappa)$ has been computed numerically by using the tables of the dilogarithm from Mitchell's paper [8] for the integrand and Simpson's rule for the integration.

For applications in physical problems, accuracy to three decimals seems to be sufficient. A table of the function $\operatorname{Le}^{*}(\alpha, \kappa)$ for $|\alpha| \leqslant 50 \quad(\Delta \alpha=1)$ and $2 \leqslant|\kappa| \leqslant$ $10(\Delta \kappa=1), 0<|\kappa| \leqslant 1 \quad(\Delta \kappa=0.1)$ is given at the end of this paper.

In Fig. 2 the function $\operatorname{Le}^{*}(\alpha, \kappa)$ is represented as a surface in the three-dimensional space over the $\alpha, \kappa$-plane. Only the part for $\kappa<0$ is shown. It can be seen that $\mathrm{Le}^{*}(\alpha, \kappa)$ possesses a sharp maximum in the vicinity of the point $(\alpha \approx 7, \kappa \approx$ $-0.3)$. For $-0.3 \leqq k<0$ the function decreases very steeply to negative values. For positive $\kappa$ the function has a similar behavior. Notice that $\lim _{\kappa \rightarrow 0} \operatorname{Le}^{*}(\alpha, \kappa)=-\infty$.

7. Conclusion. The evaluation of the integrals given by (1) has been reduced to tabulated functions. In some cases the above-mentioned table of $\operatorname{Le}^{*}(\alpha, \kappa)$ is not useable for the evaluation of $\operatorname{Le}(A, B, \gamma)$, because it is not detailed enough for interpolation in the region of very steep changes, or when $|\alpha|>50$ and $|\kappa|>10$. Then the function $\operatorname{Le}(A, B, \gamma)$ can be computed by a direct numerical integration in (16) which 


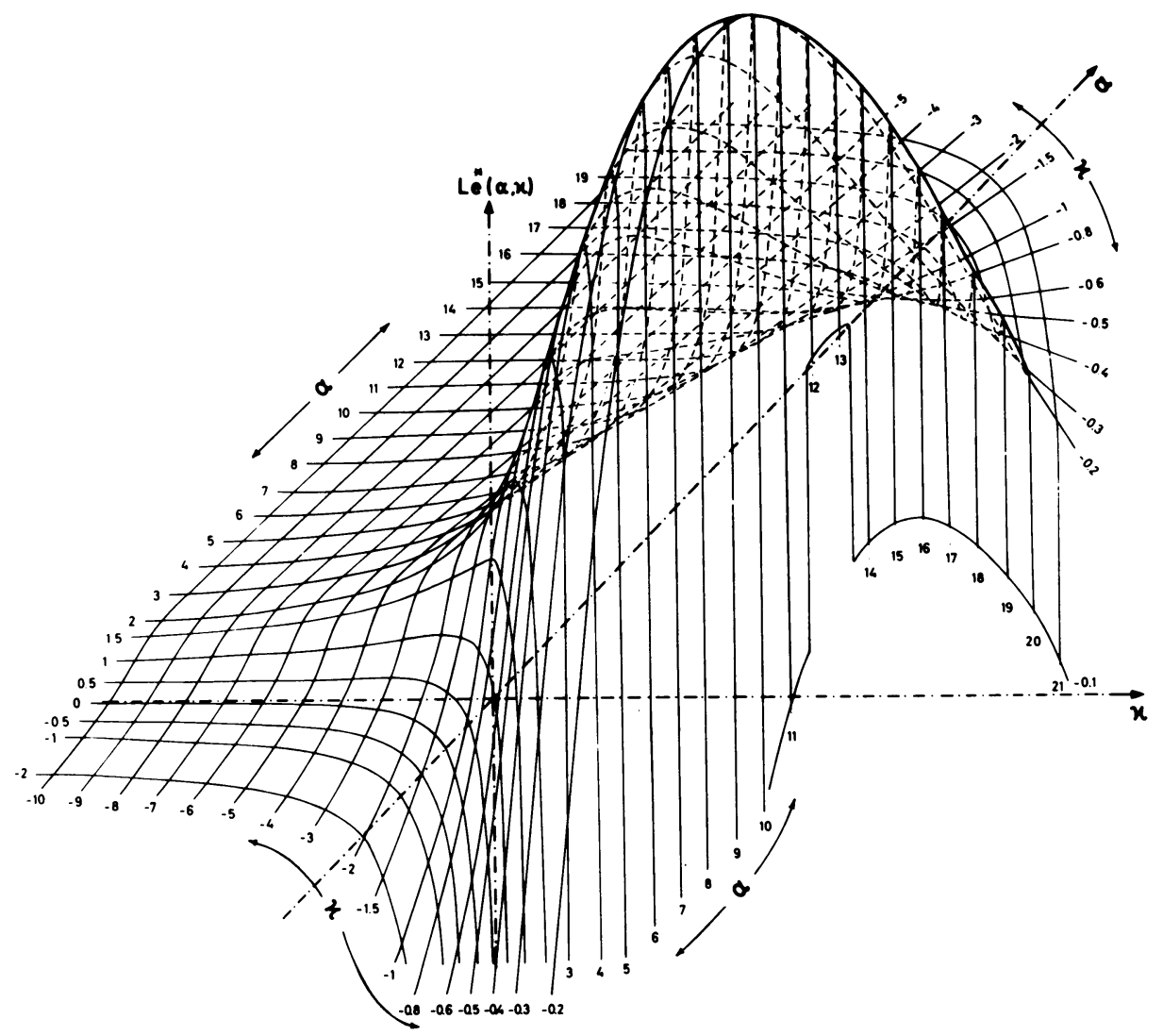

FIGURE 2. Surface over the $\alpha, \kappa$-plane described by the function $\mathrm{Le}^{*}(\alpha, \kappa), \kappa<0$.

is quite simple because the singularities on the integration path in (1) have been avoided by the previous treatment.

Acknowledgement. The author wishes-to thank Miss Hanna Tettenborn for computer programming and Mrs. Christine Degendorfer for preparing the manuscript.

Max-Planck-Institut für Physik and Astrophysik

8 München 40,

Föhringer Ring 6, Germany

1. M. ABRAMOWITZ \& I. A. STEGUN (Editors), Handbook of Mathematical Functions, With Formulas, Graphs and Mathematical Tables, Dover, New York, 1966, p. 228. MR 34 \#8606.

2. W. GRÖBNER \& N. HOFREITER (Editors), Integraltafel. Teil 1 : Unbestimmte Integrale, Springer-Verlag, Vienna, 1961, p. 113. MR 24 \#B537.

3. H. C. van de HULST, "Scattering in a planetary atmosphere," Astrophys. J., v. 107, 1948, pp. 220-246. MR 10, 151;855.

4. J. KADLEC, "Theory of internal photoemission in sandwich structures," Physics Reports. (To appear.)

5. V. KOURGANOFF \& I. W. BUSBRIDGE, Basic Methods in Transfer Problems, Clarendon Press, Oxford, 1952, p. 253. MR 14, 879.

6. L. LEWIN, Dilogarithms and Associated Functions, Macdonald, London, 1958, pp. 1-32. MR 21 \#264.

7. Y. L. LUKE, The Special Functions and Their Approximations. Vols. 1, 2, Math. in Sci. and Engrg., vol. 53, Academic Press, New York, 1969. MR 39 \# 3039; 40 \#2909.

8. K. MITCHELL, "Tables of the function $\int_{0}^{z}-\log |1-y| / y d y$ with an account of some properties of this and related functions," Philos. Mag., v. (7) 40, 1949, pp. 351-368. MR 10, 741. 
$\operatorname{Le}^{*}(\alpha, \kappa)$

\begin{tabular}{|c|c|c|c|c|c|c|c|c|c|}
\hline$\kappa$ & -10.0 & -7.0 & -9.0 & $-? .0$ & -5.0 & -5.0 & -4.0 & -3.0 & -2.0 \\
\hline $\begin{array}{r}0.0 \\
-1.0 \\
-2.0 \\
-3.0 \\
-4.0 \\
-5.0 \\
-6.0 \\
-7.0 \\
-8.0 \\
-9.0 \\
-10.0 \\
-11.0 \\
-12.0 \\
-13.0 \\
-14.0 \\
-15.0 \\
-16.0 \\
-17.0 \\
-18.0 \\
-19.0 \\
-20.0 \\
-21.0 \\
-22.0 \\
-23.0 \\
-24.0 \\
-25.0 \\
-26.0 \\
-27.0 \\
-28.0 \\
-29.0 \\
-30.0 \\
-31.0 \\
-32.0 \\
-33.0 \\
-34.0 \\
-35.0 \\
-36.0 \\
-37.0 \\
-38.0 \\
-39.0 \\
-40.0 \\
-41.0 \\
-42.0 \\
-43.0 \\
-44.0 \\
-45.0 \\
-46.0 \\
-47.0 \\
-48.0 \\
-49.0 \\
-50.0\end{array}$ & $\begin{array}{l}-0.009 \\
-0.099 \\
-0.150 \\
-0.199 \\
-0.242 \\
-0.280 \\
-0.314 \\
-0.345 \\
-0.373 \\
-0.400 \\
-0.424 \\
-0.448 \\
-0.469 \\
-0.470 \\
-0.510 \\
-0.525 \\
-0.547 \\
-0.565 \\
-0.581 \\
-0.597 \\
-0.613 \\
-0.629 \\
-0.542 \\
-0.656 \\
-0.670 \\
-0.693 \\
-0.696 \\
-0.709 \\
-0.721 \\
-0.733 \\
-0.744 \\
-0.756 \\
-0.767 \\
-0.778 \\
-0.788 \\
-0.799 \\
-0.809 \\
-0.819 \\
-0.825 \\
-0.938 \\
-0.848 \\
-0.857 \\
-0.866 \\
-0.875 \\
-0.884 \\
-0.892 \\
-0.901 \\
-0.709 \\
-0.917 \\
-0.925 \\
-0.933\end{array}$ & $\begin{array}{l}-0.012 \\
-0.100 \\
-0.16 ? \\
-0.222 \\
-0.269 \\
-0.311 \\
-0.349 \\
-0.383 \\
-0.414 \\
-0.444 \\
-0.471 \\
-0.497 \\
-0.521 \\
-0.544 \\
-0.566 \\
-0.587 \\
-0.607 \\
-0.626 \\
-0.545 \\
-0.663 \\
-0.690 \\
-0.657 \\
-0.713 \\
-0.728 \\
-0.743 \\
-0.758 \\
-0.772 \\
-0.786 \\
-0.800 \\
-0.813 \\
-0.826 \\
-0.839 \\
-0.351 \\
-0.353 \\
-0.875 \\
-0.886 \\
-0.397 \\
-0.908 \\
-0.910 \\
-0.930 \\
-0.940 \\
-0.951 \\
-0.361 \\
-0.070 \\
-0.980 \\
-0.390 \\
-0.999 \\
-1.008 \\
-1.013 \\
-1.025 \\
-1.033\end{array}$ & $\begin{array}{l}-0.015 \\
-0.113 \\
-0.138 \\
-1.050 \\
-0.393 \\
-0.351 \\
-0.392 \\
-0.431 \\
-0.465 \\
-0.499 \\
-0.530 \\
-0.557 \\
-0.586 \\
-0.512 \\
-0.630 \\
-0.061 \\
-0.683 \\
-0.704 \\
-0.725 \\
-0.745 \\
-0.764 \\
-0.783 \\
-0.801 \\
-0.817 \\
-0.836 \\
-0.852 \\
-0.363 \\
-0.884 \\
-0.897 \\
-0.914 \\
-0.928 \\
-0.942 \\
-0.056 \\
-0.970 \\
-0.383 \\
-0.996 \\
-1.009 \\
-1.021 \\
-1.033 \\
-1.045 \\
-1.057 \\
-1.068 \\
-1.083 \\
-1.001 \\
-1.102 \\
-1.112 \\
-1.123 \\
-1.133 \\
-1.143 \\
-1.153 \\
-1.103\end{array}$ & 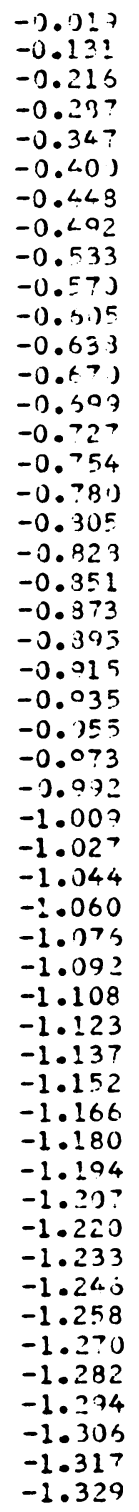 & $\begin{array}{l}-0.023 \\
-0.156 \\
-1.254 \\
-1.0336 \\
-0.406 \\
-1.468 \\
-0.524 \\
-0.575 \\
-0.522 \\
-0.566 \\
-0.797 \\
-1.0745 \\
-0.782 \\
-1.310 \\
-0.849 \\
-0.880 \\
-1.910 \\
-0.939 \\
-1.965 \\
-0.993 \\
-1.319 \\
-1.044 \\
-1.068 \\
-1.091 \\
-1.113 \\
-1.135 \\
-1.157 \\
-1.178 \\
-1.198 \\
-1.213 \\
-1.237 \\
-1.256 \\
-1.274 \\
-1.292 \\
-1.309 \\
-1.327 \\
-1.343 \\
-1.360 \\
-1.376 \\
-1.392 \\
-1.403 \\
-1.423 \\
-1.438 \\
-1.453 \\
-1.467 \\
-1.481 \\
-1.476 \\
-1.509 \\
-1.523 \\
-1.536 \\
-1.547\end{array}$ & $\begin{array}{l}-1.037 \\
-0.191 \\
-0.309 \\
-0.406 \\
-0.490 \\
-0.564 \\
-0.631 \\
-0.692 \\
-1.743 \\
-0.800 \\
-0.849 \\
-10.895 \\
-0.939 \\
-1.981 \\
-1.019 \\
-1.1557 \\
-1.093 \\
-1.127 \\
-1.160 \\
-1.192 \\
-1.223 \\
-1.253 \\
-1.282 \\
-1.310 \\
-1.337 \\
-1.363 \\
-1.389 \\
-1.413 \\
-1.438 \\
-1.461 \\
-1.484 \\
-1.507 \\
-1.529 \\
-1.550 \\
-1.572 \\
-1.592 \\
-1.612 \\
-1.632 \\
-1.651 \\
-1.670 \\
-1.689 \\
-1.707 \\
-1.726 \\
-1.743 \\
-1.761 \\
-1.778 \\
-1.795 \\
-1.811 \\
-1.823 \\
-1.843 \\
-1.859\end{array}$ & $\begin{array}{l}-0.055 \\
-0.246 \\
-1) .392 \\
-0.313 \\
-0.617 \\
-1.7) 9 \\
-0.792 \\
-0.868 \\
-1.938 \\
-1.004 \\
-1.064 \\
-1.122 \\
-1.176 \\
-1.227 \\
-1.276 \\
-1.323 \\
-1.368 \\
-1.411 \\
-1.452 \\
-1.492 \\
-1.530 \\
-1.568 \\
-1.634 \\
-1.638 \\
-1.672 \\
-1.705 \\
-1.737 \\
-1.768 \\
-1.798 \\
-1.828 \\
-1.857 \\
-1.885 \\
-1.912 \\
-1.939 \\
-1.965 \\
-1.991 \\
-2.016 \\
-2.041 \\
-2.065 \\
-2.089 \\
-2.112 \\
-2.135 \\
-2.158 \\
-2.180 \\
-2.201 \\
-2.223 \\
-2.244 \\
-2.264 \\
-2.285 \\
-2.305 \\
-2.325\end{array}$ & $\begin{array}{l}-0.098 \\
-0.345 \\
-0.536 \\
-0.695 \\
-0.833 \\
-0.955 \\
-1.065 \\
-1.166 \\
-1.258 \\
-1.345 \\
-1.426 \\
-1.502 \\
-1.574 \\
-1.642 \\
-1.707 \\
-1.769 \\
-1.829 \\
-1.386 \\
-1.941 \\
-1.993 \\
-2.045 \\
-2.094 \\
-2.142 \\
-2.188 \\
-2.233 \\
-2.277 \\
-2.319 \\
-2.360 \\
-2.401 \\
-2.440 \\
-2.478 \\
-2.516 \\
-2.552 \\
-2.588 \\
-2.623 \\
-2.657 \\
-2.691 \\
-2.724 \\
-2.756 \\
-2.788 \\
-2.919 \\
-2.849 \\
-2.879 \\
-2.908 \\
-2.937 \\
-2.966 \\
-2.994 \\
-3.021 \\
-3.049 \\
-3.075 \\
-3.101\end{array}$ & $\begin{array}{l}-0.209 \\
-0.566 \\
-0.845 \\
-1.078 \\
-1.280 \\
-1.460 \\
-1.623 \\
-1.772 \\
-1.909 \\
-2.037 \\
-2.157 \\
-2.270 \\
-2.377 \\
-2.479 \\
-2.576 \\
-2.668 \\
-2.757 \\
-2.842 \\
-2.924 \\
-3.003 \\
-3.079 \\
-3.153 \\
-3.224 \\
-3.293 \\
-3.360 \\
-3.425 \\
-3.489 \\
-3.551 \\
-3.611 \\
-3.669 \\
-3.727 \\
-3.783 \\
-3.837 \\
-3.891 \\
-3.943 \\
-3.994 \\
-4.044 \\
-4.093 \\
-4.142 \\
-4.189 \\
-4.235 \\
-4.281 \\
-4.326 \\
-4.370 \\
-4.413 \\
-4.455 \\
-4.497 \\
-4.539 \\
-4.579 \\
-4.619 \\
-4.658\end{array}$ \\
\hline
\end{tabular}


Le* $(\alpha, k)$

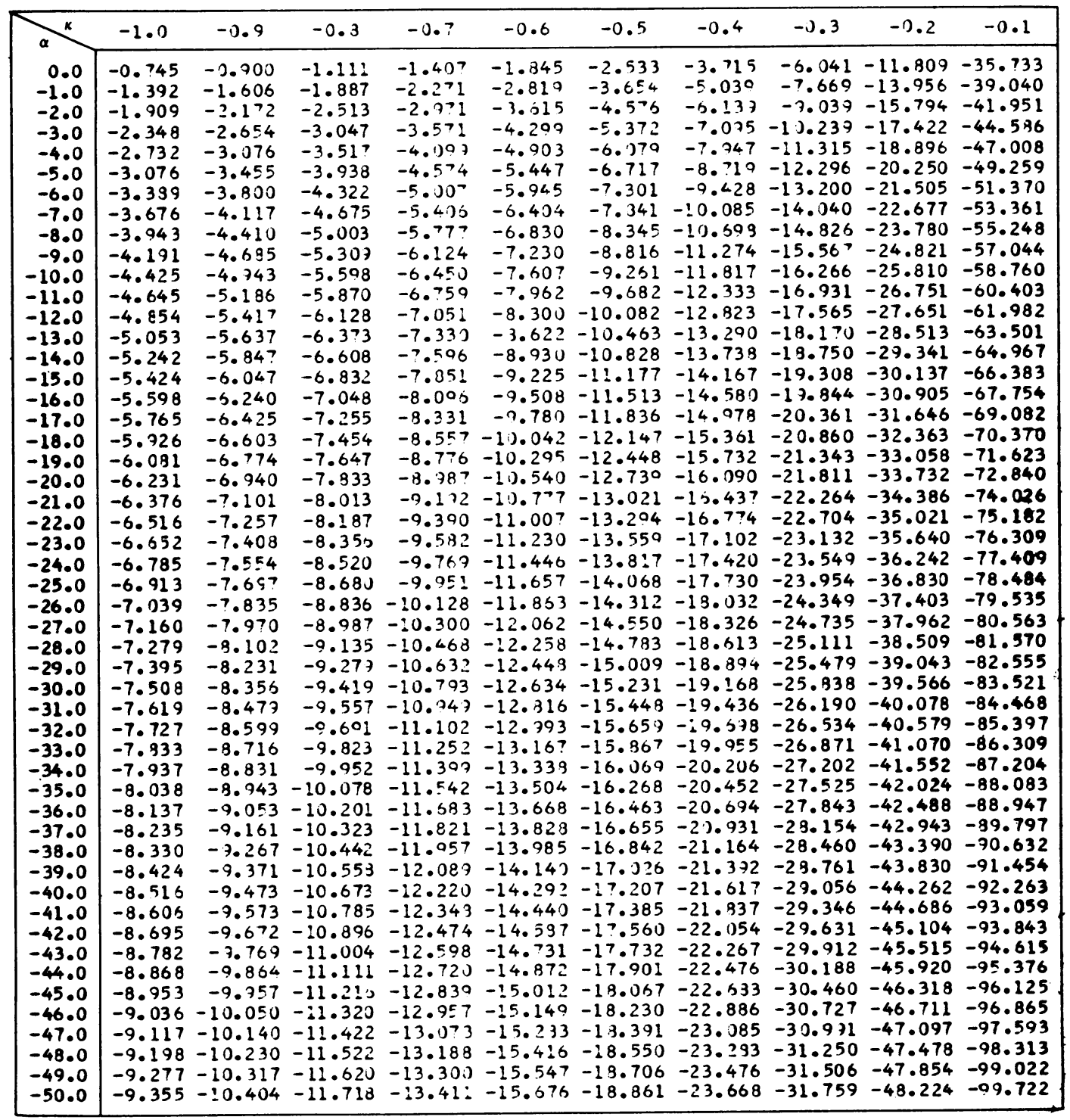


$\operatorname{Le}^{*}(\alpha, \kappa)$

\begin{tabular}{|c|c|c|c|c|c|c|c|c|c|c|}
\hline & 0.1 & 0.2 & 0.3 & 0.4 & 0.5 & $c \cdot t$ & 0.7 & C. 8 & C. G & 1.0 \\
\hline $\begin{array}{l}-2 \\
-2 \\
-2 \\
-2 \\
-30 \\
-31 \\
-3 \\
-3 \\
-3 \\
-3 \\
-3 \\
-3 \\
-3 \\
-3 \\
-4 \\
-4 \\
-42\end{array}$ & $\begin{array}{r}9.399 \\
8.083 \\
6.223 \\
4.013 \\
1.573 \\
-2.986 \\
-5.485 \\
-8.056 \\
-10.665 \\
-13.287 \\
-15.902 \\
-18.494 \\
-21.057 \\
-23.580 \\
-26.056 \\
-28.480 \\
-30.850 \\
-33.162 \\
-35.417 \\
-37.611 \\
-39.748 \\
-41.825 \\
-43.845 \\
-45.809 \\
-47.717 \\
-49.571 \\
-51.373 \\
-53.124 \\
-54.825 \\
-56.479 \\
-58.088 \\
-59.652 \\
-61.173 \\
-62.654 \\
-64.096 \\
-65.500 \\
-66.868 \\
-68.200 \\
-69.500 \\
-70.768 \\
-72.005 \\
-73.212 \\
-74.392 \\
-75.544 \\
-76.671 \\
-77.772 \\
-78.850 \\
-79.905 \\
-80.938 \\
-81.949 \\
-82.940\end{array}$ & $\begin{array}{r}6.955 \\
5.286 \\
3.284 \\
1.145 \\
-1.102 \\
-3.231 \\
-5.279 \\
-7.286 \\
-9.183 \\
-10.994 \\
-12.691 \\
-14.305 \\
-15.831 \\
-17.274 \\
-18.639 \\
-19.933 \\
-21.159 \\
-22.323 \\
-23.431 \\
-24.485 \\
-25.492 \\
-26.453 \\
-27.374 \\
-28.257 \\
-29.106 \\
-29.921 \\
-30.708 \\
-31.466 \\
-32.199 \\
-32.908 \\
-33.545 \\
-34.261 \\
-40.528 \\
-41.023 \\
-41.507 \\
-41.983 \\
-42.449 \\
-42.906 \\
-43.359 .790 \\
-34.536 \\
-36.147 \\
-36.742 \\
-37.322 \\
-37.838 \\
-38.440 \\
-3907\end{array}$ & $\begin{array}{r}5.14 C \\
3.414 \\
1.530 \\
-0.348 \\
-2.132 \\
-3.8 C 2 \\
-5.347 \\
-6.775 \\
-8.067 \\
-9.297 \\
-10.414 \\
-11.448 \\
-12.409 \\
-13.336 \\
-14.146 \\
-14.935 \\
-15.679 \\
-16.362 \\
-17.050 \\
-17.685 \\
-18.293 \\
-18.875 \\
-19.433 \\
-19.969 \\
-20.485 \\
-20.984 \\
-21.466 \\
-21.932 \\
-22.384 \\
-22.823 \\
-23.249 \\
-23.664 \\
-24.067 \\
-24.461 \\
-24.844 \\
-25.219 \\
-25.585 \\
-25.942 \\
-26.292 \\
-26.635 \\
-26.970 \\
-29.29 .9996 \\
-27.621 \\
-27.937 \\
-28.247 \\
-28.552 \\
-28.851 \\
-29.144 \\
-29.93\end{array}$ & $\begin{array}{r}3.720 \\
2.232 \\
0.530 \\
-1.050 \\
-2.405 \\
-3.796 \\
-4.952 \\
-6.011 \\
-6.059 \\
-7.823 \\
-8.608 \\
-9.334 \\
-10.006 \\
-10.632 \\
-11.219 \\
-11.772 \\
-12.205 \\
-12.791 \\
-13.263 \\
-13.715 \\
-14.147 \\
-14.562 \\
-14.952 \\
-15.347 \\
-15.719 \\
-16.078 \\
-16.425 \\
-11.764 \\
-17.092 \\
-17.411 \\
-17.722 \\
-18.024 \\
-18.310 \\
-19.607 \\
-18.387 \\
-19.162 \\
-19.430 \\
-19.632 \\
-19.949 \\
-20.201 \\
-20.447 \\
-20.589 \\
-20.926 \\
-21.159 \\
-21.337 \\
-21.612 \\
-21.832 \\
-22.049 \\
-22.252 \\
-22.472 \\
-22.678\end{array}$ & 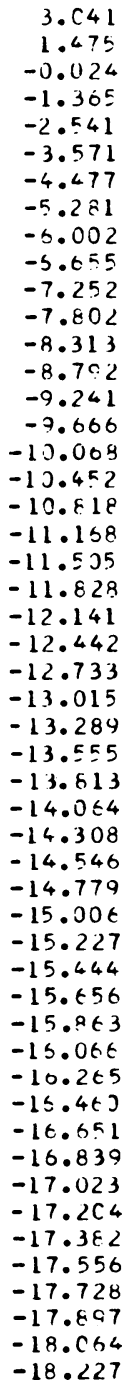 & 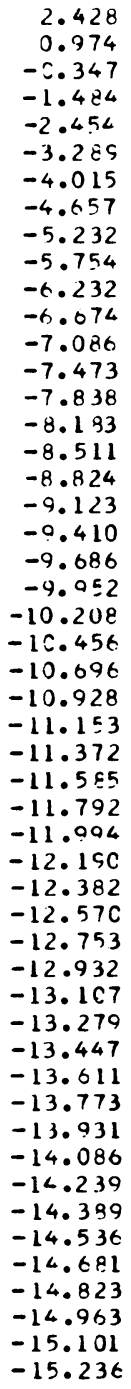 & 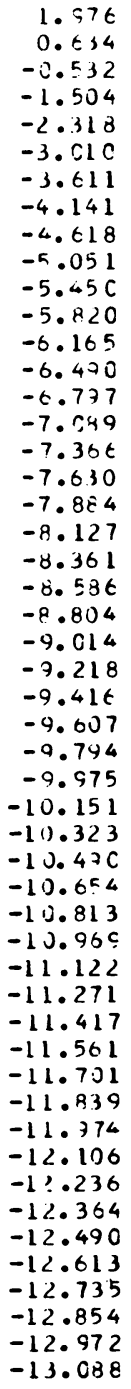 & 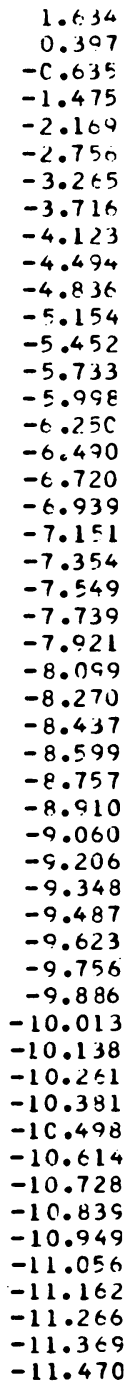 & $\begin{array}{l}1.360 \\
0.229 \\
-0.689 \\
-1.422 \\
-2.023 \\
-2.531 \\
-2.973 \\
-3.365 \\
-3.719 \\
-4.044 \\
-4.344 \\
-4.623 \\
-4.885 \\
-5.132 \\
-5.366 \\
-5.588 \\
-5.800 \\
-6.002 \\
-6.196 \\
-6.383 \\
-6.562 \\
-6.735 \\
-6.903 \\
-7.064 \\
-7.221 \\
-7.373 \\
-7.521 \\
-7.664 \\
-7.803 \\
-7.939 \\
-8.072 \\
-10.028 \\
-10.119 \\
-10.208 \\
-8.301 \\
-9.9\end{array}$ & $\begin{array}{l}1.161 \\
0.109 \\
-0.713 \\
-1.360 \\
-1.888 \\
-2.334 \\
-2.724 \\
-3.071 \\
-3.385 \\
-3.674 \\
-3.941 \\
-4.190 \\
-4.424 \\
-4.644 \\
-4.853 \\
-5.052 \\
-5.242 \\
-5.423 \\
-5.597 \\
-5.764 \\
-5.924 \\
-6.079 \\
-6.229 \\
-6.374 \\
-6.515 \\
-6.651 \\
-6.784 \\
-6.912 \\
-7.037 \\
-7.159 \\
-7.278 \\
-7.394 \\
-7.507 \\
-7.618 \\
-7.726 \\
-7.832 \\
-7.935 \\
-8.037 \\
-8.136 \\
-8.233 \\
-8.329 \\
-8.423 \\
-8.515 \\
-8.605 \\
-8.694 \\
-8.781 \\
-8.867 \\
-8.751 \\
-9.034 \\
-9.116 \\
-9.196\end{array}$ \\
\hline
\end{tabular}


$\mathbf{L e}^{*}(\alpha, \kappa)$

\begin{tabular}{|c|c|c|c|c|c|c|c|c|c|}
\hline & 2.0 & 3.0 & 4.0 & 5.0 & 6.0 & 7.0 & $0 . J$ & 9.0 & 10.0 \\
\hline 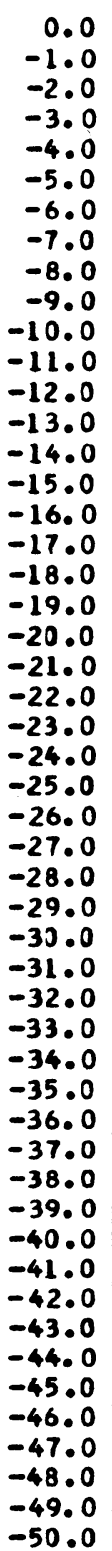 & $\begin{array}{l}0.330 \\
-0.199 \\
-0.562 \\
-0.843 \\
-1.077 \\
-1.280 \\
-1.460 \\
-1.622 \\
-1.771 \\
-1.909 \\
-2.037 \\
-2.157 \\
-2.270 \\
-2.377 \\
-2.479 \\
-2.576 \\
-2.668 \\
-2.757 \\
-2.842 \\
-2.924 \\
-3.002 \\
-3.079 \\
-3.152 \\
-3.223 \\
-3.293 \\
-3.360 \\
-3.425 \\
-3.488 \\
-3.550 \\
-3.610 \\
-3.669 \\
-3.726 \\
-3.782 \\
-3.837 \\
-3.890 \\
-3.942 \\
-3.994 \\
-4.044 \\
-4.093 \\
-4.141 \\
-4.188 \\
-4.235 \\
-4.280 \\
-4.325 \\
-4.369 \\
-4.412 \\
-4.455 \\
-4.497 \\
-4.538 \\
-4.579 \\
-4.618\end{array}$ & $\begin{array}{l}0.141 \\
-0.187 \\
-0.413 \\
-0.592 \\
-0.743 \\
-0.875 \\
-0.993 \\
-1.099 \\
-1.197 \\
-1.288 \\
-1.372 \\
-1.451 \\
-1.526 \\
-1.597 \\
-1.664 \\
-1.728 \\
-1.789 \\
-1.348 \\
-1.904 \\
-1.958 \\
-2.010 \\
-2.061 \\
-2.110 \\
-2.157 \\
-2.203 \\
-2.247 \\
-2.291 \\
-2.333 \\
-2.374 \\
-2.414 \\
-2.452 \\
-2.490 \\
-2.528 \\
-2.564 \\
-2.599 \\
-2.634 \\
-2.668 \\
-2.701 \\
-2.734 \\
-2.766 \\
-2.798 \\
-2.828 \\
-2.859 \\
-2.888 \\
-2.918 \\
-2.946 \\
-2.975 \\
-3.003 \\
-3.030 \\
-3.057 \\
-3.083\end{array}$ & $\begin{array}{l}0.075 \\
-0.159 \\
-0.323 \\
-0.455 \\
-1.567 \\
-0.664 \\
-0.752 \\
-0.831 \\
-0.904 \\
-0.972 \\
-1.034 \\
-1.094 \\
-1.149 \\
-1.202 \\
-1.252 \\
-1.300 \\
-1.346 \\
-1.389 \\
-1.432 \\
-1.472 \\
-1.511 \\
-1.549 \\
-1.585 \\
-1.621 \\
-1.655 \\
-1.688 \\
-1.721 \\
-1.752 \\
-1.783 \\
-1.813 \\
-1.842 \\
-1.870 \\
-1.858 \\
-1.925 \\
-1.952 \\
-1.978 \\
-2.003 \\
-2.028 \\
-2.053 \\
-2.077 \\
-2.100 \\
-2.123 \\
-2.146 \\
-2.168 \\
-2.193 \\
-2.212 \\
-2.233 \\
-2.254 \\
-2.274 \\
-2.295 \\
-2.314\end{array}$ & $\begin{array}{l}0.045 \\
-0.135 \\
-0.265 \\
-0.367 \\
-0.453 \\
-0.535 \\
-0.005 \\
-0.668 \\
-0.725 \\
-0.780 \\
-0.830 \\
-0.877 \\
-1.522 \\
-0.954 \\
-1.004 \\
-1.1042 \\
-1.077 \\
-1.114 \\
-1.147 \\
-1.180 \\
-1.211 \\
-1.241 \\
-1.270 \\
-1.298 \\
-1.326 \\
-1.352 \\
-1.378 \\
-1.403 \\
-1.428 \\
-1.452 \\
-1.475 \\
-1.499 \\
-1.520 \\
-1.542 \\
-1.563 \\
-1.584 \\
-1.604 \\
-1.624 \\
-1.643 \\
-1.653 \\
-1.682 \\
-1.700 \\
-1.718 \\
-1.736 \\
-1.754 \\
-1.771 \\
-1.788 \\
-1.804 \\
-1.821 \\
-1.837 \\
-1.853\end{array}$ & $\begin{array}{l}1.031 \\
-0.117 \\
-0.224 \\
-0.310 \\
-0.384 \\
-0.448 \\
-0.506 \\
-0.559 \\
-0.607 \\
-0.652 \\
-0.693 \\
-0.733 \\
-0.770 \\
-0.805 \\
-0.838 \\
-0.870 \\
-0.900 \\
-0.929 \\
-0.957 \\
-0.984 \\
-1.010 \\
-1.035 \\
-1.060 \\
-1.083 \\
-1.106 \\
-1.128 \\
-1.150 \\
-1.171 \\
-1.191 \\
-1.211 \\
-1.230 \\
-1.249 \\
-1.268 \\
-1.286 \\
-1.303 \\
-1.321 \\
-1.338 \\
-1.354 \\
-1.371 \\
-1.387 \\
-1.402 \\
-1.418 \\
-1.433 \\
-1.448 \\
-1.462 \\
-1.477 \\
-1.491 \\
-1.505 \\
-1.518 \\
-1.532 \\
-1.545\end{array}$ & $\begin{array}{l}0.022 \\
-0.103 \\
-0.174 \\
-0.258 \\
-0.331 \\
-0.386 \\
-0.435 \\
-0.480 \\
-0.522 \\
-0.56 C \\
-0.596 \\
-0.629 \\
-0.661 \\
-0.591 \\
-0.719 \\
-0.747 \\
-0.773 \\
-0.798 \\
-0.822 \\
-0.845 \\
-0.867 \\
-0.888 \\
-0.909 \\
-0.929 \\
-0.949 \\
-0.968 \\
-0.986 \\
-1.004 \\
-1.022 \\
-1.039 \\
-1.056 \\
-1.072 \\
-1.088 \\
-1.103 \\
-1.118 \\
-1.133 \\
-1.148 \\
-1.162 \\
-1.176 \\
-1.190 \\
-1.203 \\
-1.216 \\
-1.229 \\
-1.242 \\
-1.254 \\
-1.267 \\
-1.279 \\
-1.291 \\
-1.302 \\
-1.314 \\
-1.325\end{array}$ & $\begin{array}{l}0.017 \\
-0.092 \\
-0.171 \\
-0.236 \\
-0.291 \\
-0.335 \\
-0.382 \\
-0.421 \\
-0.457 \\
-0.491 \\
-0.522 \\
-0.552 \\
-0.579 \\
-0.605 \\
-0.630 \\
-0.654 \\
-0.677 \\
-0.699 \\
-0.720 \\
-0.740 \\
-0.760 \\
-0.778 \\
-0.797 \\
-0.814 \\
-0.831 \\
-0.848 \\
-0.864 \\
-0.880 \\
-0.895 \\
-0.910 \\
-0.925 \\
-0.939 \\
-0.953 \\
-0.966 \\
-0.980 \\
-0.993 \\
-1.005 \\
-1.018 \\
-1.030 \\
-1.042 \\
-1.054 \\
-1.065 \\
-1.077 \\
-1.088 \\
-1.099 \\
-1.109 \\
-1.120 \\
-1.130 \\
-1.141 \\
-1.151 \\
-1.161\end{array}$ & $\begin{array}{l}0.013 \\
-0.083 \\
-0.153 \\
-0.210 \\
-0.259 \\
-0.302 \\
-0.340 \\
-0.375 \\
-0.408 \\
-0.437 \\
-0.465 \\
-0.491 \\
-0.516 \\
-0.539 \\
-0.561 \\
-0.583 \\
-0.603 \\
-0.622 \\
-0.641 \\
-0.659 \\
-0.676 \\
-0.693 \\
-0.709 \\
-0.725 \\
-0.740 \\
-0.755 \\
-0.769 \\
-0.783 \\
-0.797 \\
-0.810 \\
-0.823 \\
-0.836 \\
-0.848 \\
-0.860 \\
-0.872 \\
-0.883 \\
-0.895 \\
-0.906 \\
-0.917 \\
-0.927 \\
-0.938 \\
-0.948 \\
-0.958 \\
-0.968 \\
-0.978 \\
-0.987 \\
-0.997 \\
-1.006 \\
-1.015 \\
-1.0244 \\
-1.033\end{array}$ & $\begin{array}{l}0.010 \\
-0.076 \\
-0.139 \\
-0.190 \\
-0.234 \\
-0.273 \\
-0.307 \\
-0.339 \\
-0.368 \\
-0.394 \\
-0.420 \\
-0.443 \\
-0.465 \\
-0.486 \\
-0.506 \\
-0.525 \\
-0.544 \\
-0.561 \\
-0.578 \\
-0.594 \\
-0.610 \\
-0.625 \\
-0.639 \\
-0.654 \\
-0.667 \\
-0.681 \\
-0.694 \\
-0.706 \\
-0.718 \\
-0.730 \\
-0.742 \\
-0.753 \\
-0.765 \\
-0.776 \\
-0.786 \\
-0.797 \\
-0.807 \\
-0.817 \\
-0.827 \\
-0.836 \\
-0.846 \\
-0.8555 \\
-0.864 \\
-0.873 \\
-0.882 \\
-0.890 \\
-0.899 \\
-0.907 \\
-0.915 \\
-0.923 \\
-0.931\end{array}$ \\
\hline
\end{tabular}


$\operatorname{Le}^{*}(\alpha, k)$

\begin{tabular}{|c|c|c|c|c|c|c|c|c|c|}
\hline & 0.0 & 9.0 & -8.0 & -7.0 & -6.0 & -5.0 & -4.0 & -3.0 & -2.0 \\
\hline 49.0 & $\begin{array}{l}-0.440 \\
-0.432\end{array}$ & $\begin{array}{l}-0.498 \\
-0.479\end{array}$ & $\begin{array}{l}-0.548 \\
-0.538\end{array}$ & $\begin{array}{l}-0.625 \\
-0.614\end{array}$ & $\begin{array}{l}-0.729 \\
-0.716\end{array}$ & $\begin{array}{l}-0.874 \\
-0.8 \leq 8\end{array}$ & $\begin{array}{l}-1.071 \\
-1.071\end{array}$ & $\begin{array}{l}-1.452 \\
-1.426\end{array}$ & $\begin{array}{l}-2.171 \\
-2.132\end{array}$ \\
\hline & -0.424 & $-0.4 ? 0$ & -0.528 & -0.0 .33 & $-1.70 ?$ & -0.842 & -1.051 & -1.399 & \\
\hline 47.0 & -0.416 & -0.461 & -0.513 & -0.591 & -0.689 & -0.826 & $-1.03:$ & -1.372 & -2.051 \\
\hline 46.0 & -0.407 & -0.452 & $-0.50 ?$ & -0.577 & -0.675 & $-0.8 .9 ?$ & -1.010 & -1.344 & -2.010 \\
\hline 45.0 & -0.399 & -0.442 & $-0.49 ?$ & -0.567 & $-0.66:$ & -0.792 & -10.999 & -1.316 & -1.968 \\
\hline 44.0 & -0.390 & -0.433 & -0.435 & -0.555 & -0.647 & -0.775 & $-1) .968$ & -1.288 & -1.925 \\
\hline 43.0 & -0.382 & $-1) .423$ & -0.475 & -0.543 & -0.632 & -0.753 & -0.946 & -1.259 & -1.881 \\
\hline 42.0 & -0.373 & -3.414 & -0.464 & -0.530 & -0.618 & $-0.74 !$ & -0.924 & -1.230 & -1.837 \\
\hline 41.0 & -0.354 &.- .404 & $-0.4 \equiv 3$ & $-0.51 ?$ & -1.0 .603 & -0.723 & -0.002 & -1.200 & -1.792 \\
\hline 40.0 & -0.355 & $-0.3 \div 3$ & -0.442 & -0.504 & -0.593 & -.0 .734 & -0.873 & -1.167 & -1.746 \\
\hline 39.i) & -0.345 & -3.393 & $-1) .43 J$ & -3.401 & -0.572 & -0.686 & -10.856 & -1.130 & -1.700 \\
\hline 38.0 & -0.336 & -3.373 & -0.418 & $-0.4>3$ & $-0.55 j$ & -0.667 & -0.832 & -1.107 & -1.652 \\
\hline 37.0 & -0.326 & -0.262 & -0.406 & -0.464 & -0.540 & -0.649 & -1.909 & -1.075 & -1.504 \\
\hline 36.0 & $-0.31 \epsilon$ & $-1.3=1$ & -0.394 & -0.45)$. & -0.524 & -0.628 & -0.784 & -1.042 & -1.554 \\
\hline 35.0 & $-0.3: 56$ & -0.34)$. & -0.382 & -0.436 & $-0.5) ?$ & $-3.6) 9$ & -1.759 & -1.008 & -1.506 \\
\hline 34.0 & -0.276 & -3.223 & -0.369 & $-0.42 i$ & $-0.49 J$ & -0.587 & $-1) .733$ & -0.374 & -1.453 \\
\hline 33.0 & -0.296 & $-3.3: 7$ & -10.350 & -0.400 & $-0.4 ? 3$ & $-0.5 t ?$ & -0.707 & -0.030 & -1.400 \\
\hline 32.1$)$ & -0.275 & -1.305 & $-0.34 \geq$ & $-0.3 .7:$ & -0.455 & -1.545 & -1.580 & -3.904 & -1.346 \\
\hline 31.10 & -0.254 & $-1.2 ; 3$ & -0.323 & $-10.3=$ & -0.437 & -0.523 & -0.653 & -0.867 & -1.292 \\
\hline 30.0 & -0.253 & -3.290 & -3.115 & -0.357 & -1.413 & -) .501$. & -0.625 & $-i) .830$ & -1.235 \\
\hline 29.0 & -3.241 & -3.208 & -0.301 & -0.343 & -0.329 & -0.4 .73 & -0.576 & -0.792 & -1.178 \\
\hline 29.J & $-0.23 c$ & $-1.25 \xi$ & -0.230 & $-0.32 t$ & -1.380 & -0.455 & -0.567 & -0.753 & -1.119 \\
\hline 27.0 & -0.218 & $-0.24:$ & $-0.27:$ & $-3.3 i)$ & $-1.35 i$ & -3.431 & -.0 .537 & -0.713 & -1.059 \\
\hline 26.1$)$ & -1.235 & -1.2 .23 & -0.255 & -0.202 & -3.347 & $-0.40 t$ & -0.506 & -.0 .672 & -0.797 \\
\hline 25.0 & -0.173 & $-3.2 ! 4$ & -0.241 & -0.274 & $-1.3: 13$ & -0.331 & -0.475 & -0.629 & -0.033 \\
\hline 24.0 & -0.180 & -0.137 & -0.2 .24 & -0.253 & -3.23 & -3 & $-j$ & -1.586 & -0.368 \\
\hline 23. 0 & -0.155 & $-1.1:: 4$ & $-0.20 ?$ & $-0.23 t$ & -0.275 & $-0.32^{\circ}$ & -0.407 & -0.541 & -0.801 \\
\hline 22.5 & & $-1.2 \div 3$ & $-0 . \vdots 3)$ & -0.216 & $-0.25 ?$ & & -1 & -3.496 & -0.732 \\
\hline 21.0 & -0.135 & $-1.1=4$ & $-0 .: ? ?$ & $-0 .: 0 i$ & -0.223 & -0. & -3 & -3 & 60 \\
\hline 20.0 & -0.224 & -1.137 & $-1) .: 54$ & $-0.1>$ & $-3.2 i) 4$ & -0.244 & -1.303 & -1.300 & -0.587 \\
\hline 19.0 & & $-1 .: \therefore i$ & $-J .23 j$ & $-1 .: 5$ & $-1 .: \div 7$ & & $-\jmath$ & -0.349 & -.0 .510 \\
\hline 19.:) & $-i) \cdot C 32$ & -3.103 & $-0.1: 3$ & -0.132 & -0.553 & -0.182 & $-0.22 h$ & $-i)$. & -0.432 \\
\hline 17.0 & -0. & -נ.) & , , & $(-0.1 .01$ & $-0.12 \%$ & $-0 .: 50$ & -3.185 & -9.243 & -0.350 \\
\hline 16.0 & & -0.1 & $-0.0 ? 3$ & $-0 . J B=$ & -0.073 & -0 & & & -0.265 \\
\hline 15.0 & $-1) .043$ & -3.) \rightarrow$ ? & $-0.1) 53$ & $(-1)).(1)$ & -0.060 & -0.032 & -0. & $-1) .128$ & 177 \\
\hline 14..) & -0.3 & $-) .12 ?$ & -0.031 & $-10.03 \cdot 4$ & -1.032 & -0.1445 & $-j$. & -1) 63$. & -0.086 \\
\hline 13.0 & & לניט.ט-ט- & -0.007 & -0.007 & -0.008 & & & & 0.010 \\
\hline 12.0 & .0 .014 & ).J!' & $0.0 \div 3$ & $0.102:$ & 0.025 & ) & 0.042 & 0.062 & 0.110 \\
\hline 11.0 & 0 & ) .0 . & D. . 54 & 0.1551 & .06) & 0.)^{\top 3}$ & ). .94 & 0.131 & 0.215 \\
\hline 10.11 & & & 0.0 & & 0.0 & & 0. & & 0.325 \\
\hline 0.0 & & .) \times 9$ & 0.377 & $0.1: \therefore$ & $1 .: 34$ & 0.262 & 0.2105 & 7.280 & 0.440 \\
\hline 8.1$)$ & $0.1: 3 ?$ & 1). 214 & 0.129 & 1). $-<3$ & 0.173 & $0.2: 0$ & 1.253 & 3.360 & 0.562 \\
\hline 7.0 & & 3.142 & $0.100)$ & & ) .215 & & 0. & & 0.690 \\
\hline 6.1 & 0.154 & ) $.1 ?$ & 0.133 & 0.??? & .0 .253 & ). 313 & 1.395 & 3.534 & 0.823 \\
\hline 5.1 & $0 .: 92$ & ) .202 & 0.228 & $0.2 t$ : & .305 & 1.0 .353 & ) .463 & ) .525 & 0.950 \\
\hline 4.J) & $0 . ? 10$ & 0.233 & 0.262 & $0.30 J$ & 0.351 & & & J.7 & 1.090 \\
\hline 3.0 & 0.235 & $3.26 i$ & $0 . ? 74$ & .333 & 1.373 & $0 .+? 2$ & & 0.791 & $1.17 \varepsilon$ \\
\hline 2.0 & 0.247 & .27 & $0 . ? 08$ & $0.35 !$ & 0.4193 & 0.436 & 0.500 & 0.778 & 1.076 \\
\hline 1.0 & 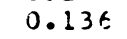 & $0.1 \div 3$ & & , & $0.2 .3:$ & & 0.264 & 1.310 & $0.35 ?$ \\
\hline 0.0 & -0.009 & $-0.0: 2$ & -0.015 & $-0.0: 3$ & $-1.02 \geq 0$ & $-0.03 ?$ & -0.056 & -0.098 & -0.209 \\
\hline
\end{tabular}


$\operatorname{Le}^{*}(\alpha, \kappa)$

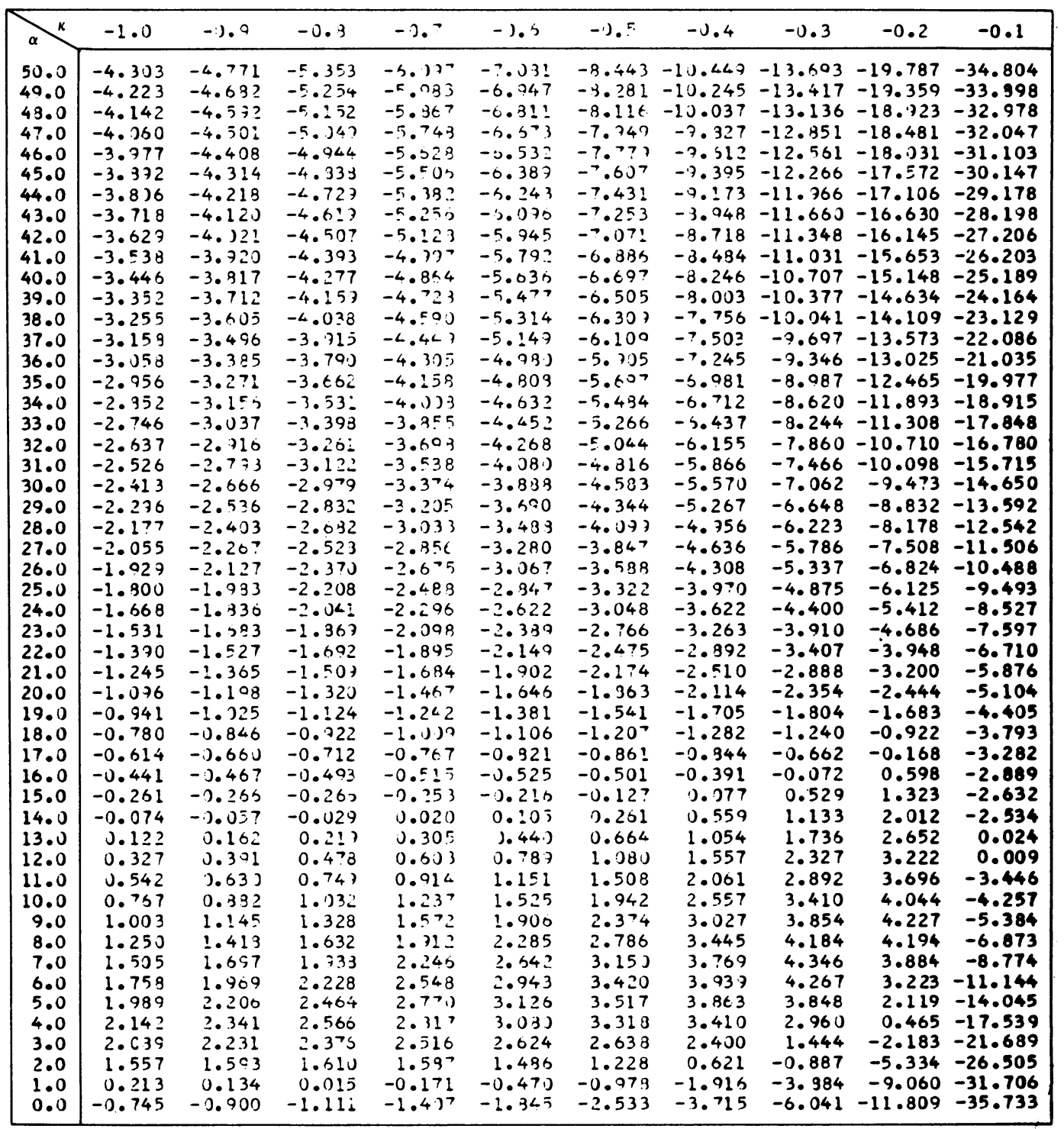


$\operatorname{Le}^{*}(\alpha, \kappa)$

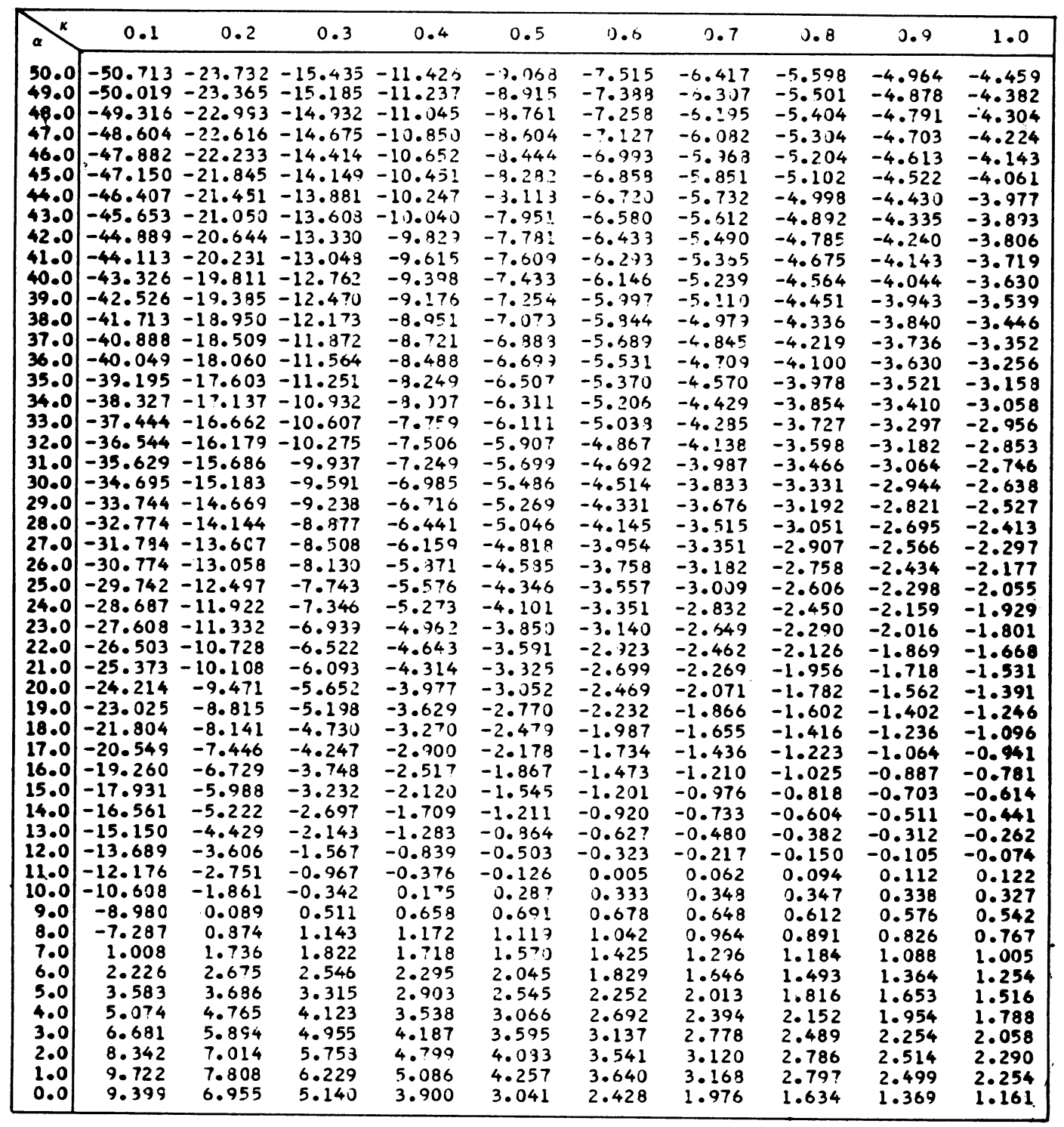


$\operatorname{Le}^{*}(\alpha, \kappa)$

\begin{tabular}{|c|c|c|c|c|c|c|c|c|c|}
\hline & 2.0 & 3.0 & 4.0 & 5.0 & 6.0 & 7.0 & 8.0 & 9.0 & 10.0 \\
\hline $\begin{array}{r}50.0 \\
49.0 \\
48.0 \\
47.0 \\
46.0 \\
45.0 \\
44.0 \\
43.0 \\
42.0 \\
41.0 \\
40.0 \\
39.0 \\
38.0 \\
37.0 \\
36.0 \\
35.0 \\
34.0 \\
33.0 \\
32.0 \\
31.0 \\
30.0 \\
29.0 \\
28.0 \\
27.0 \\
26.0 \\
25.0 \\
24.0 \\
23.0 \\
22.0 \\
21.0 \\
20.0 \\
19.0 \\
18.0 \\
17.0 \\
16.0 \\
15.0 \\
14.0 \\
13.0 \\
12.0 \\
11.0 \\
10.0 \\
9.0 \\
8.0 \\
7.0 \\
6.0 \\
5.0 \\
4.0 \\
3.0 \\
2.0 \\
1.0 \\
0.0\end{array}$ & $\begin{array}{l}-2.211 \\
-2.172 \\
-2.132 \\
-2.092 \\
-2.051 \\
-2.010 \\
-1.968 \\
-1.925 \\
-1.882 \\
-1.837 \\
-1.793 \\
-1.747 \\
-1.700 \\
-1.653 \\
-1.604 \\
-1.555 \\
-1.504 \\
-1.453 \\
-1.400 \\
-1.347 \\
-1.292 \\
-1.236 \\
-1.178 \\
-1.119 \\
-1.059 \\
-0.997 \\
-0.933 \\
-0.868 \\
-0.801 \\
-0.732 \\
-0.660 \\
-0.587 \\
-0.511 \\
-0.432 \\
-0.350 \\
-0.265 \\
-0.177 \\
-0.086 \\
0.010 \\
0.110 \\
0.215 \\
0.324 \\
0.440 \\
0.562 \\
0.690 \\
0.924 \\
0.962 \\
1.096 \\
1.201 \\
1.104 \\
0.330\end{array}$ & $\begin{array}{l}-1.470 \\
-1.443 \\
-1.417 \\
-1.390 \\
-1.363 \\
-1.335 \\
-1.307 \\
-1.279 \\
-1.250 \\
-1.220 \\
-1.190 \\
-1.159 \\
-1.128 \\
-1.053 \\
-1.064 \\
-1.031 \\
-10.997 \\
-0.953 \\
-0.928 \\
-0.892 \\
-0.855 \\
-0.817 \\
-0.779 \\
-0.740 \\
-0.699 \\
-0.658 \\
-0.615 \\
-0.571 \\
-0.526 \\
-0.480 \\
-0.432 \\
-0.383 \\
-0.332 \\
-0.279 \\
-0.224 \\
-0.168 \\
-0.108 \\
-0.047 \\
0.017 \\
0.084 \\
0.155 \\
0.229 \\
0.306 \\
0.388 \\
0.474 \\
0.564 \\
0.656 \\
0.746 \\
0.811 \\
0.713 \\
0.141\end{array}$ & $\begin{array}{l}-1.101 \\
-1.081 \\
-1.061 \\
-1.041 \\
-1.021 \\
-1.000 \\
-0.979 \\
-0.957 \\
-0.936 \\
-0.913 \\
-0.891 \\
-0.868 \\
-0.844 \\
-0.320 \\
-0.796 \\
-0.771 \\
-0.746 \\
-0.720 \\
-0.694 \\
-0.667 \\
-0.639 \\
-0.611 \\
-0.582 \\
-0.552 \\
-0.522 \\
-0.491 \\
-0.459 \\
-0.426 \\
-0.392 \\
-0.357 \\
-0.321 \\
-0.284 \\
-0.246 \\
-0.206 \\
-0.165 \\
-0.122 \\
-0.078 \\
-0.031 \\
0.017 \\
0.068 \\
0.121 \\
0.176 \\
0.235 \\
0.296 \\
0.361 \\
0.427 \\
0.498 \\
0.565 \\
0.612 \\
0.521 \\
0.075\end{array}$ & $\begin{array}{l}-0.880 \\
-0.864 \\
-0.849 \\
-0.832 \\
-0.816 \\
-0.797 \\
-0.782 \\
-0.765 \\
-0.748 \\
-0.730 \\
-0.712 \\
-0.693 \\
-0.675 \\
-0.655 \\
-0.636 \\
-0.616 \\
-0.596 \\
-0.575 \\
-0.554 \\
-0.532 \\
-0.510 \\
-0.488 \\
-0.464 \\
-0.441 \\
-0.416 \\
-0.391 \\
-0.366 \\
-0.339 \\
-0.312 \\
-0.284 \\
-0.256 \\
-0.226 \\
-0.195 \\
-0.163 \\
-0.130 \\
-0.096 \\
-0.060 \\
-0.023 \\
0.016 \\
0.056 \\
0.099 \\
0.143 \\
0.191 \\
0.240 \\
0.292 \\
0.346 \\
0.401 \\
0.454 \\
0.491 \\
0.408 \\
0.046\end{array}$ & $\begin{array}{l}-0.733 \\
-0.720 \\
-0.707 \\
-0.693 \\
-0.680 \\
-0.666 \\
-0.652 \\
-0.637 \\
-0.623 \\
-0.608 \\
-0.593 \\
-0.578 \\
-0.562 \\
-0.546 \\
-0.530 \\
-0.513 \\
-0.496 \\
-0.479 \\
-0.461 \\
-0.443 \\
-0.425 \\
-0.406 \\
-0.387 \\
-0.367 \\
-0.346 \\
-0.326 \\
-0.304 \\
-0.282 \\
-0.260 \\
-0.236 \\
-0.212 \\
-0.187 \\
-0.162 \\
-0.135 \\
-0.108 \\
-0.079 \\
-0.049 \\
-0.018 \\
0.014 \\
0.049 \\
0.083 \\
0.121 \\
0.167 \\
0.201 \\
0.245 \\
0.290 \\
0.336 \\
0.380 \\
0.410 \\
0.334 \\
0.031\end{array}$ & $\begin{array}{l}-0.628 \\
-0.617 \\
-0.606 \\
-0.594 \\
-0.583 \\
-0.571 \\
-0.559 \\
-0.546 \\
-0.534 \\
-0.521 \\
-0.508 \\
-0.495 \\
-0.481 \\
-0.468 \\
-0.454 \\
-0.440 \\
-0.425 \\
-0.410 \\
-0.395 \\
-0.380 \\
-0.364 \\
-0.348 \\
-0.331 \\
-0.314 \\
-0.297 \\
-0.279 \\
-0.260 \\
-0.242 \\
-0.222 \\
-0.202 \\
-0.182 \\
-0.160 \\
-0.138 \\
-0.115 \\
-0.092 \\
-0.067 \\
-0.042 \\
-0.015 \\
0.013 \\
0.042 \\
0.072 \\
0.104 \\
0.138 \\
0.173 \\
0.211 \\
0.250 \\
0.289 \\
0.327 \\
0.352 \\
0.282 \\
0.022\end{array}$ & $\begin{array}{l}-0.550 \\
-0.540 \\
-0.530 \\
-0.520 \\
-0.510 \\
-0.500 \\
-0.489 \\
-0.478 \\
-0.467 \\
-0.456 \\
-0.445 \\
-0.433 \\
-0.421 \\
-0.409 \\
-0.397 \\
-0.385 \\
-0.372 \\
-0.359 \\
-0.346 \\
-0.332 \\
-0.318 \\
-0.304 \\
-0.290 \\
-0.275 \\
-0.260 \\
-0.244 \\
-0.228 \\
-0.211 \\
-0.194 \\
-0.177 \\
-0.159 \\
-0.140 \\
-0.121 \\
-0.101 \\
-0.081 \\
-0.058 \\
-0.036 \\
-0.013 \\
0.012 \\
0.037 \\
0.064 \\
0.092 \\
0.121 \\
0.152 \\
0.185 \\
0.219 \\
0.254 \\
0.287 \\
0.308 \\
0.243 \\
0.017\end{array}$ & $\begin{array}{l}-0.490 \\
-0.481 \\
-0.472 \\
-0.463 \\
-0.454 \\
-0.444 \\
-0.435 \\
-0.425 \\
-0.416 \\
-0.406 \\
-0.396 \\
-0.385 \\
-0.375 \\
-0.364 \\
-0.353 \\
-0.342 \\
-0.331 \\
-0.319 \\
-0.308 \\
-0.296 \\
-0.283 \\
-0.271 \\
-0.258 \\
-0.244 \\
-0.231 \\
-0.217 \\
-0.203 \\
-0.188 \\
-0.173 \\
-0.157 \\
-0.141 \\
-0.124 \\
-0.107 \\
-0.089 \\
-0.071 \\
-0.052 \\
-0.032 \\
-0.011 \\
0.011 \\
0.033 \\
0.057 \\
0.082 \\
0.108 \\
0.136 \\
0.165 \\
0.195 \\
0.226 \\
0.256 \\
0.275 \\
0.214 \\
0.013\end{array}$ & $\begin{array}{l}-0.441 \\
-0.433 \\
-0.425 \\
-0.417 \\
-0.409 \\
-0.401 \\
-0.392 \\
-0.383 \\
-0.375 \\
-0.366 \\
-0.357 \\
-0.347 \\
-0.338 \\
-0.328 \\
-0.318 \\
-0.308 \\
-0.298 \\
-0.288 \\
-0.277 \\
-0.266 \\
-0.255 \\
-0.244 \\
-0.232 \\
-0.220 \\
-0.208 \\
-0.195 \\
-0.182 \\
-0.169 \\
-0.155 \\
-0.141 \\
-0.127 \\
-0.112 \\
-0.096 \\
-0.080 \\
-0.064 \\
-0.046 \\
-0.028 \\
-0.010 \\
0.010 \\
0.030 \\
0.052 \\
0.074 \\
0.098 \\
0.123 \\
0.149 \\
0.177 \\
0.204 \\
0.231 \\
0.248 \\
0.191 \\
0.010\end{array}$ \\
\hline
\end{tabular}

